\title{
Hurwitz Equivalence in Tuples of Generalized Quaternion Groups and Dihedral Groups
}

\author{
Xiang-dong Hou \\ Department of Mathematics \\ University of South Florida, Tampa, FL 33620 \\ xhou@math.usf .edu \\ Submitted: Apr 6, 2008; Accepted: May 29, 2008; Published: Jun 13, 2008 \\ Mathematics Subject Classifications: 20F36, 20F05
}

\begin{abstract}
Let $Q_{2^{m}}$ be the generalized quaternion group of order $2^{m}$ and $D_{N}$ the dihedral group of order $2 N$. We classify the orbits in $Q_{2^{m}}^{n}$ and $D_{p^{m}}^{n}$ ( $p$ prime) under the Hurwitz action.
\end{abstract}

\section{The Hurwitz Action}

Let $G$ be a group. For $a, b \in G$, let $a^{b}=b^{-1} a b$ and ${ }^{b} a=b a b^{-1}$. The Hurwitz action on $G^{n}(n \geq 2)$ is an action of the $n$-string braid group $B_{n}$ on $G^{n}$. Recall that $B_{n}$ is given by the presentation

$$
B_{n}=\left\langle\sigma_{1}, \ldots, \sigma_{n-1}\left|\sigma_{i} \sigma_{j}=\sigma_{j} \sigma_{i},\right| i-j \mid>2 ; \sigma_{i} \sigma_{i+1} \sigma_{i}=\sigma_{i+1} \sigma_{i} \sigma_{i+1}, 1 \leq i \leq n-2\right\rangle .
$$

The action of $\sigma_{i}$ on $G^{n}$ is defined by

$$
\sigma_{i}\left(a_{1}, \ldots, a_{n}\right)=\left(a_{1}, \ldots, a_{i-1}, a_{i+1}, a_{i}^{a_{i+1}}, a_{i+2}, \ldots, a_{n}\right),
$$

where $\left(a_{1}, \ldots, a_{n}\right) \in G^{n}$. Note that

$$
\sigma_{i}^{-1}\left(a_{1}, \ldots, a_{n}\right)=\left(a_{1}, \ldots, a_{i-1},{ }^{a_{i}} a_{i+1}, a_{i}, a_{i+2}, \ldots, a_{n}\right) .
$$

An action by $\sigma_{i}$ or $\sigma_{i}^{-1}$ on $G^{n}$ is called a Hurwitz move. Two tuples $\left(a_{1}, \ldots, a_{n}\right)$, $\left(b_{1}, \ldots, b_{n}\right) \in G^{n}$ are called (Hurwitz) equivalent, denoted as $\left(a_{1}, \ldots, a_{n}\right) \sim\left(b_{1}, \ldots, b_{n}\right)$, if they are in the same $B_{n}$-orbit. The (Hurwitz) equivalence class of $\left(a_{1}, \ldots, a_{n}\right) \in G^{n}$, i.e., the $B_{n}$-orbit of $\left(a_{1}, \ldots, a_{n}\right)$, is denoted by $\left[a_{1}, \ldots, a_{n}\right]$.

If $G$ is a nonabelian group, in general, the $B_{n}$-orbits in $G^{n}$ are not known. In [1], Ben-Itzhak and Teicher determined all $B_{n}$-orbits in $S_{m}^{n}$ represented by $\left(t_{1}, \ldots, t_{n}\right)$, where $S_{m}$ is the symmetric group, each $t_{i}$ is a transposition and $t_{1} \cdots t_{n}=1$. It is obvious that if 
$a_{1}, \ldots, a_{n} \in G$ generate a finite subgroup, then the $B_{n^{-}}$orbit of $\left(a_{1}, \ldots, a_{n}\right)$ in $G^{n}$ is finite. It has been proved that if $s_{1}, \ldots, s_{n} \in \mathrm{GL}\left(\mathbb{R}^{n}\right)$ are reflections such that the $B_{n}$-orbit of $\left(s_{1}, \ldots, s_{n}\right)$ is finite, then the group generated by $s_{1}, \ldots, s_{n}$ is finite; see [2] and [3].

It is natural to ask which types of nonabelian group $G$ allow complete determination of the $B_{n}$-orbits in $G^{n}$. In this paper, we show that when $G$ is the generalized quaternion group $Q_{2^{m}}$ or the dihedral group $D_{p^{m}}$ of order $2 p^{m}$, where $p$ is a prime, the answer to the above question is affirmative.

\section{The Generalized Quaternion Group}

Let $m \geq 2$. The generalized quaternion group $Q_{2^{m}}$ of order $2^{m}$ is given by the presentation

$$
Q_{2^{m}}=\left\langle\alpha, \beta \mid \alpha^{2^{m-1}}=1, \alpha^{2^{m-2}}=\beta^{2}, \beta \alpha \beta^{-1}=\alpha^{-1}\right\rangle .
$$

Each element of $Q_{2^{m}}$ can be uniquely written as $\alpha^{i} \beta^{j}$, where $0 \leq i<2^{m-1}$ and $0 \leq j \leq 1$. We have

$$
\begin{gathered}
\left(\alpha^{i} \beta^{j}\right)^{\alpha^{k} \beta^{l}}=\alpha^{(-1)^{l}(i-2 k j)} \beta^{j}, \\
\alpha^{i} \beta^{j}\left(\alpha^{k} \beta^{l}\right)=\alpha^{(-1)^{j} k+2 i l} \beta^{l} .
\end{gathered}
$$

Thus in $Q_{2^{m}}^{n}$, a Hurwitz move gives one of the following equivalences:

$$
\begin{gathered}
\left(\cdots, \alpha^{i} \beta^{j}, \alpha^{k} \beta^{l}, \cdots\right) \sim\left(\cdots, \alpha^{k} \beta^{l}, \alpha^{(-1)^{l}(i-2 k j)} \beta^{j}, \cdots\right), \\
\left(\cdots, \alpha^{i} \beta^{j}, \alpha^{k} \beta^{l}, \cdots\right) \sim\left(\cdots, \alpha^{(-1)^{j} k+2 i l} \beta^{l}, \alpha^{i} \beta^{j}, \cdots\right) .
\end{gathered}
$$

For easier reading, we rewrite the above equivalences, omitting the $\ldots$ 's, with $(j, l)=$ $(0,0),(0,1),(1,0)$ and $(1,1)$ respectively.

$$
\begin{gathered}
\left(\alpha^{i}, \alpha^{k}\right) \sim\left(\alpha^{k}, \alpha^{i}\right), \\
\left\{\begin{array}{l}
\left(\alpha^{i}, \alpha^{k} \beta\right) \sim\left(\alpha^{k} \beta, \alpha^{-i}\right), \\
\left(\alpha^{i}, \alpha^{k} \beta\right) \sim\left(\alpha^{k+2 i} \beta, \alpha^{i}\right),
\end{array}\right. \\
\left\{\begin{array}{l}
\left(\alpha^{i} \beta, \alpha^{k}\right) \sim\left(\alpha^{k}, \alpha^{i-2 k} \beta\right), \\
\left(\alpha^{i} \beta, \alpha^{k}\right) \sim\left(\alpha^{-k}, \alpha^{i} \beta\right),
\end{array}\right. \\
\left\{\begin{array}{l}
\left(\alpha^{i} \beta, \alpha^{k} \beta\right) \sim\left(\alpha^{k} \beta, \alpha^{-i+2 k} \beta\right)=\left(\alpha^{i+(k-i)} \beta, \alpha^{k+(k-i)} \beta\right), \\
\left(\alpha^{i} \beta, \alpha^{k} \beta\right) \sim\left(\alpha^{-k+2 i} \beta, \alpha^{i} \beta\right)=\left(\alpha^{i-(k-i)} \beta, \alpha^{k-(k-i)} \beta\right) .
\end{array}\right.
\end{gathered}
$$

Lemma 2.1. (i) $\left(\alpha^{i}, \alpha^{j} \beta\right) \sim\left(\alpha^{-i}, \alpha^{j+2 i} \beta\right)$ for all $i, j \in \mathbb{Z}$.

(ii) $\left(\alpha^{i} \beta, \alpha^{j} \beta\right) \sim\left(\alpha^{i+k(j-i)} \beta, \alpha^{j+k(j-i)} \beta\right)$ for all $i, j, k \in \mathbb{Z}$.

(iii) Let $\tau, \nu, e, f \in \mathbb{Z}$ such that $0 \leq \nu \leq m-2$ and $e \not \equiv f(\bmod 2)$. Then for every $g \in \mathbb{Z}$

$$
\left(\alpha^{\tau+2^{\nu} e} \beta, \alpha^{\tau+2^{\nu} f} \beta\right) \sim\left(\alpha^{\tau+2^{\nu}(e+g)} \beta, \alpha^{\tau+2^{\nu}(f+g)} \beta\right) .
$$


Proof. (i) We have

$$
\begin{array}{rlrl}
\left(\alpha^{i}, \alpha^{j} \beta\right) & \sim\left(\alpha^{j} \beta, \alpha^{-i}\right) & & \text { (the first eq. of }(2.4)) \\
& \sim\left(\alpha^{-i}, \alpha^{j+2 i} \beta\right) & \quad \text { (the first eq. of }(2.5)) .
\end{array}
$$

(ii) follows from $(2.6)$.

(iii) In (ii) let $i=\tau+2^{\nu} e, j=\tau+2^{\nu} f$ and choose $k \in \mathbb{Z}$ such that $k 2^{\nu}(f-e) \equiv g 2^{\nu}$ $\left(\bmod 2^{m-1}\right)$.

\section{$3 \quad B_{n}$-Orbits in $Q_{2^{m}}^{n}$}

Let $G$ be a group. For $\boldsymbol{a}=\left(a_{1}, \ldots, a_{n}\right) \in G^{n}$, define $\pi(\boldsymbol{a})=a_{1} \cdots a_{n} \in G$. $\pi(\boldsymbol{a})$ is an invariant of the Hurwitz action on $G^{n}$.

For $\boldsymbol{a}=\left(\alpha^{i_{1}} \beta^{j_{1}}, \ldots, \alpha^{i_{n}} \beta^{j_{n}}\right) \in Q_{2^{m}}^{n}$, where $0 \leq i_{k}<2^{m-1}$ and $0 \leq j_{k} \leq 1$, let

$$
\begin{gathered}
\Lambda(\boldsymbol{a})=\text { the multi set }\left\{\min \left\{i_{k}, 2^{m-1}-i_{k}\right\}: j_{k}=0\right\}, \\
\Gamma(\boldsymbol{a})=\left\{i_{k}: j_{k}=1\right\} .
\end{gathered}
$$

For example, if $\boldsymbol{a}=\left(\alpha^{3} \beta, \alpha^{4} \beta, \alpha^{3} \beta, \alpha \beta\right), \boldsymbol{b}=\left(\alpha^{6}, \alpha \beta, 1, \alpha^{2}\right) \in Q_{2^{4}}^{4}$, then $\Lambda(\boldsymbol{a})=\varnothing$, $\Lambda(\boldsymbol{b})=\{0,2,2\}, \Gamma(\boldsymbol{a})=\{1,3,4\}, \Gamma(\boldsymbol{b})=\{1\} . \Lambda(\boldsymbol{a})$ is an invariant of the Hurwitz action on $Q_{2^{m}}^{n}$. In fact, it is easy to see that $\Lambda(\boldsymbol{a})$ is invariant under each of the Hurwitz moves in $(2.3)-(2.6)$.

To determine the $B_{n^{-}}$orbits in $Q_{2^{m}}^{n}$, we first partition $Q_{2^{m}}^{n}$ into suitable subsets. Let

$$
\mathcal{A}=\left\{\boldsymbol{a} \in Q_{2^{m}}^{n}: \Gamma(\boldsymbol{a})=\varnothing\right\} .
$$

For each $1 \leq \nu \leq m-1$ and $0 \leq \tau<2^{\nu}$, let

$$
\mathcal{B}_{\nu, \tau}=\left\{\boldsymbol{a} \in Q_{2^{m}}^{n}: \min \left(\left\{\nu_{2}(i): i \in \Lambda(\boldsymbol{a})\right\} \cup\{m-2\}\right)=\nu-1, \varnothing \neq \Gamma(\boldsymbol{a}) \subset \tau+2^{\nu} \mathbb{Z}\right\},
$$

where $\nu_{2}$ is the 2-adic order. For each $0 \leq \nu \leq m-2$ and $0 \leq \tau<2^{\nu}$, let

$$
\begin{gathered}
\mathcal{C}_{\nu, \tau}=\left\{\boldsymbol{a} \in Q_{2^{m}}^{n}:\right. \\
\min \left(\left\{\nu_{2}(i): i \in \Lambda(\boldsymbol{a})\right\} \cup\{m-2\}\right) \geq \nu, \Gamma(\boldsymbol{a}) \subset \tau+2^{\nu} \mathbb{Z}, \\
\left.\exists j, j^{\prime} \in \Gamma(\boldsymbol{a}) \text { such that } \nu_{2}\left(j-j^{\prime}\right)=\nu\right\} .
\end{gathered}
$$

Then

$$
Q_{2^{m}}^{n}=\mathcal{A} \dot{\cup}\left(\bigcup_{\substack{1 \leq \nu \leq m-1 \\ 0 \leq \tau<2^{\nu}}}^{\cdot} \mathcal{B}_{\nu, \tau}\right) \dot{\cup}\left(\bigcup_{\substack{0 \leq \nu \leq m-2 \\ 0 \leq \tau<2^{\nu}}}^{\cdot} \mathcal{C}_{\nu, \tau}\right) .
$$

It is routine to check that each of $\mathcal{A}, \mathcal{B}_{\nu, \tau}, \mathcal{C}_{\nu, \tau}$ is invariant under the Hurwitz moves in $(2.3)-(2.6)$. Thus, $\mathcal{A}, \mathcal{B}_{\nu, \tau}$ and $\mathcal{C}_{\nu, \tau}$ are invariant under the Hurwitz equivalence. Therefore, to determine the $B_{n}$-orbits in $Q_{2^{m}}^{n}$, it suffices to find a set of representatives of the $B_{n}$-orbits in each of $\mathcal{A}, \mathcal{B}_{\nu, \tau}$ and $\mathcal{C}_{\nu, \tau}$. 
For $\boldsymbol{a}=\left(\alpha^{i_{1}} \beta^{j_{1}}, \ldots, \alpha^{i_{n}} \beta^{j_{n}}\right) \in \mathcal{C}_{\nu, \tau}$, where $0 \leq i_{k}<2^{m-1}$ and $0 \leq j_{k} \leq 1$, let

$$
t(\boldsymbol{a})=\mid\left\{k: j_{k}=1 \text { and } i_{k} \equiv \tau \quad\left(\bmod 2^{\nu+1}\right)\right\} \mid .
$$

We claim that $t(\boldsymbol{a})$ is an invariant under the Hurwitz equivalence. Once again, it is easy to see that $t(\boldsymbol{a})$ is invariant under the Hurwitz moves in $(2.3)-(2.6)$.

Theorem 3.1. (i) The $B_{n}$-orbits in $\mathcal{A}$ are represented by

$$
\left(\alpha^{i_{1}}, \ldots, \alpha^{i_{n}}\right)
$$

where $0 \leq i_{1} \leq \cdots \leq i_{n}<2^{m-1}$.

(ii) Let $1 \leq \nu \leq m-1$ and $0 \leq \tau<2^{\nu}$. The $B_{n}$-orbits in $\mathcal{B}_{\nu, \tau}$ are represented by

$$
\left(\alpha^{i_{1}}, \ldots, \alpha^{i_{s}}, \alpha^{\tau+2^{\nu} e} \beta, \alpha^{\tau} \beta, \ldots, \alpha^{\tau} \beta\right),
$$

where $0 \leq i_{1} \leq \cdots \leq i_{s} \leq 2^{m-2}, \min \left\{\nu_{2}\left(i_{1}\right), \ldots, \nu_{2}\left(i_{s}\right), m-2\right\}=\nu-1,0 \leq e<$ $2^{m-1-\nu}$.

(iii) Let $0 \leq \nu \leq m-2$ and $0 \leq \tau<2^{\nu}$. The $B_{n}$-orbits in $\mathcal{C}_{\nu, \tau}$ are represented by

$$
(\alpha^{i_{1}}, \ldots, \alpha^{i_{s}}, \alpha^{\tau+2^{\nu} e} \beta, \alpha^{\tau+2^{\nu}} \beta, \ldots, \alpha^{\tau+2^{\nu}} \beta, \underbrace{\alpha^{\tau} \beta, \ldots, \alpha^{\tau} \beta}_{t}),
$$

where $0 \leq i_{1} \leq \cdots \leq i_{s} \leq 2^{m-2}, \min \left\{\nu_{2}\left(i_{1}\right), \ldots, \nu_{2}\left(i_{s}\right), m-2\right\} \geq \nu, 0 \leq e<2^{m-1-\nu}$, $e \equiv 1(\bmod 2), t>0$.

Proof. (i) is obvious.

(ii) We first observe that different tuples in (3.1) have different combinations of invariants $\Lambda(\boldsymbol{a})$ and $\pi(\boldsymbol{a})$. Thus, different tuples in (3.1) are nonequivalent.

Next, we show that every $\boldsymbol{a} \in \mathcal{B}_{\nu, \tau}$ is equivalent to one of the tuples in (3.1). We may assume that

$$
\boldsymbol{a}=\left(\alpha^{i_{1}^{\prime}}, \ldots, \alpha^{i_{s-1}^{\prime}}, \alpha^{\tau+2^{\nu} e_{1}} \beta, \ldots, \alpha^{\tau+2^{\nu} e_{t}} \beta, \alpha^{j_{0}}\right),
$$

where $\nu_{2}\left(j_{0}\right)=\nu-1$. Using $(2.5)$ repeatedly, we have

$$
\begin{aligned}
& \left(\alpha^{\tau+2^{\nu} e_{1}} \beta, \ldots, \alpha^{\tau+2^{\nu} e_{t}} \beta, \alpha^{j_{0}}\right) \\
\sim & \left(\alpha^{\tau+2^{\nu} e_{1}} \beta, \ldots, \alpha^{\tau+2^{\nu} e_{t-1}} \beta, \alpha^{j_{1}}, \alpha^{\tau+2^{\nu} e_{t}^{\prime}} \beta\right) \\
\sim & \cdots \\
\sim & \left(\alpha^{j_{t}}, \alpha^{\tau+2^{\nu} e_{1}^{\prime}} \beta, \ldots, \alpha^{\tau+2^{\nu} e_{t}^{\prime}} \beta\right),
\end{aligned}
$$

where $\nu_{2}\left(j_{0}\right)=\cdots=\nu_{2}\left(j_{t}\right)=\nu-1, e_{1}^{\prime}, \ldots, e_{t-1}^{\prime}$ are even and $e_{t}^{\prime}$ is odd. Using Lemma 2.1 (iii) repeatedly, we have

$$
\begin{aligned}
& \left(\alpha^{\tau+2^{\nu} e_{1}^{\prime}} \beta, \ldots, \alpha^{\tau+2^{\nu} e_{t-1}^{\prime}} \beta, \alpha^{\tau+2^{\nu} e_{t}^{\prime}} \beta\right) \\
\sim & \left(\alpha^{\tau+2^{\nu} e_{1}^{\prime}} \beta, \ldots, \alpha^{\tau+2^{\nu} f_{1}} \beta, \alpha^{\tau} \beta\right) \quad\left(f_{1} \text { odd }\right) \\
\sim & \cdots \\
\sim & \left(\alpha^{\tau+2^{\nu} f_{t-1}} \beta, \alpha^{\tau} \beta, \ldots, \alpha^{\tau} \beta\right) \quad\left(f_{t-1} \text { odd }\right) .
\end{aligned}
$$


Combining (3.3) - (3.5), we have

$$
\begin{aligned}
\boldsymbol{a} & \sim\left(\alpha^{i_{1}^{\prime}}, \ldots, \alpha^{i_{s-1}^{\prime}}, \alpha^{j_{t}}, \alpha^{\tau+2^{\nu} f_{t-1}} \beta, \alpha^{\tau} \beta, \ldots, \alpha^{\tau} \beta\right) \\
& \sim\left(\alpha^{i_{1}}, \ldots, \alpha^{i_{s}}, \alpha^{\tau+2^{\nu} e} \beta, \alpha^{\tau} \beta, \ldots, \alpha^{\tau} \beta\right) \quad(\text { by Lemma } 2.1(\mathrm{i})),
\end{aligned}
$$

where $0 \leq i_{1} \leq \cdots \leq i_{s} \leq 2^{m-2}$ and $0 \leq e<2^{m-1-\nu}$.

(iii) Different tuples in (3.2) have different combinations of invariants $\Lambda(\boldsymbol{a}), t(\boldsymbol{a})$ and $\pi(\boldsymbol{a})$. Hence different tuples in (3.2) are nonequivalent.

It remains to show that every $\boldsymbol{a} \in \mathcal{C}_{\nu, \tau}$ is equivalent to one of the tuples in (3.2). We may assume that

$$
\boldsymbol{a}=\left(\alpha^{i_{1}}, \ldots, \alpha^{i_{s}}, \alpha^{\tau+2^{\nu} e_{1}} \beta, \ldots, \alpha^{\tau+2^{\nu} e_{u}} \beta, \alpha^{\tau+2^{\nu} f_{1}} \beta, \ldots, \alpha^{\tau+2^{\nu} f_{t}} \beta\right),
$$

where $0 \leq i_{1} \leq \cdots \leq i_{s} \leq 2^{m-2}, u>0, t>0, e_{1}, \ldots, e_{u}$ are odd and $f_{1}, \ldots, f_{t}$ are even. We have

$$
\begin{aligned}
& \left(\alpha^{\tau+2^{\nu} e_{1}} \beta, \ldots, \alpha^{\tau+2^{\nu} e_{u}} \beta, \alpha^{\tau+2^{\nu} f_{1}} \beta, \ldots, \alpha^{\tau+2^{\nu} f_{t}} \beta\right) \\
\sim & \left(\alpha^{\tau+2^{\nu} f_{0}^{\prime}} \beta, \alpha^{\tau+2^{\nu} e_{1}} \beta, \ldots, \alpha^{\tau+2^{\nu} e_{u}} \beta, \alpha^{\tau+2^{\nu} f_{2}} \beta, \ldots, \alpha^{\tau+2^{\nu} f_{t}} \beta\right) \quad\left(f_{0}^{\prime} \text { even }\right),
\end{aligned}
$$

where

$$
\begin{array}{rlr} 
& \left(\alpha^{\tau+2^{\nu} f_{0}^{\prime}} \beta, \alpha^{\tau+2^{\nu} e_{1}} \beta, \ldots, \alpha^{\tau+2^{\nu} e_{u}} \beta\right) & \\
\sim & \left(\alpha^{\tau+2^{\nu}} \beta, \alpha^{\tau+2^{\nu} f_{1}^{\prime}} \beta, \alpha^{\tau+2^{\nu} e_{2}} \beta, \ldots, \alpha^{\tau+2^{\nu} e_{u}} \beta\right) & \left(f_{1}^{\prime} \text { even, Lemma 2.1 (iii) }\right) \\
\sim & \cdots & \\
\sim & \left(\alpha^{\tau+2^{\nu}} \beta, \ldots, \alpha^{\tau+2^{\nu}} \beta, \alpha^{\tau+2^{\nu} f_{u}^{\prime}} \beta\right) & \left(f_{u}^{\prime} \text { even }\right) .
\end{array}
$$

Hence

$$
\begin{aligned}
& \left(\alpha^{\tau+2^{\nu} e_{1}} \beta, \ldots, \alpha^{\tau+2^{\nu} e_{u}} \beta, \alpha^{\tau+2^{\nu} f_{1}} \beta, \ldots, \alpha^{\tau+2^{\nu} f_{t}} \beta\right) \\
\sim & \left(\alpha^{\tau+2^{\nu}} \beta, \ldots, \alpha^{\tau+2^{\nu}} \beta, \alpha^{\tau+2^{\nu} f_{u}^{\prime}} \beta, \alpha^{\tau+2^{\nu} f_{2}} \beta, \ldots, \alpha^{\tau+2^{\nu} f_{t}} \beta\right) .
\end{aligned}
$$

By a similar argument,

$$
\begin{aligned}
& \left(\alpha^{\tau+2^{\nu}} \beta, \alpha^{\tau+2^{\nu} f_{u}^{\prime}} \beta, \alpha^{\tau+2^{\nu} f_{2}} \beta, \ldots, \alpha^{\tau+2^{\nu} f_{t}} \beta\right) \\
\sim & \left(\alpha^{\tau+2^{\nu} h} \beta, \alpha^{\tau} \beta, \ldots, \alpha^{\tau} \beta\right) \quad(h \text { odd }) .
\end{aligned}
$$

By (3.7) and (3.8),

$$
\begin{aligned}
& \left(\alpha^{\tau+2^{\nu} e_{1}} \beta, \ldots, \alpha^{\tau+2^{\nu} e_{u}} \beta, \alpha^{\tau+2^{\nu} f_{1}} \beta, \ldots, \alpha^{\tau+2^{\nu} f_{t}} \beta\right) \\
\sim & \left(\alpha^{\tau+2^{\nu}} \beta, \ldots, \alpha^{\tau+2^{\nu}} \beta, \alpha^{\tau+2^{\nu} h} \beta, \alpha^{\tau} \beta, \ldots, \alpha^{\tau} \beta\right) \\
\sim & \left(\alpha^{\tau+2^{\nu} e} \beta, \alpha^{\tau+2^{\nu}} \beta, \ldots, \alpha^{\tau+2^{\nu}} \beta, \alpha^{\tau} \beta, \ldots, \alpha^{\tau} \beta\right) \quad(e \text { odd }) .
\end{aligned}
$$

Combining (3.6) and (3.9), we see that $\alpha$ is equivalent to the tuple in (3.2).

Theorem 3.1 has an immediate corollary. 
Corollary 3.2. (i) $\boldsymbol{a}, \boldsymbol{b} \in \mathcal{A}$ are equivalent $\Leftrightarrow \boldsymbol{a}$ is a permutation of $\boldsymbol{b}$.

(ii) $\boldsymbol{a}, \boldsymbol{b} \in \mathcal{B}_{\nu, \tau}$ are equivalent $\Leftrightarrow \Lambda(\boldsymbol{a})=\Lambda(\boldsymbol{b})$ and $\pi(\boldsymbol{a})=\pi(\boldsymbol{b})$.

(iii) $\boldsymbol{a}, \boldsymbol{b} \in \mathcal{C}_{\nu, \tau}$ are equivalent $\Leftrightarrow \Lambda(\boldsymbol{a})=\Lambda(\boldsymbol{b}), t(\boldsymbol{a})=t(\boldsymbol{b})$ and $\pi(\boldsymbol{a})=\pi(\boldsymbol{b})$.

Theorem 3.1 and Corollary 3.2 allow us to compute the number of $B_{n}$-orbits in $\mathbb{Q}_{2^{m}}^{n}$ and the cardinality of each $B_{n}$-orbit.

Corollary 3.3. The total number of equivalence classes in $Q_{2^{m}}^{n}$ is

$$
\left|Q_{2^{m}}^{n} / \sim\right|=\left(\begin{array}{c}
n+2^{m-1}-1 \\
n
\end{array}\right)+2^{m-1}\left(\begin{array}{c}
n+2^{m-2} \\
n-1
\end{array}\right)+2^{m-2} \sum_{\nu=0}^{m-2}\left(\begin{array}{c}
n+2^{m-2-\nu} \\
n-2
\end{array}\right) .
$$

Proof. By Theorem 3.1 (i),

$$
|\mathcal{A} / \sim|=\left(\begin{array}{c}
n+2^{m-1}-1 \\
n
\end{array}\right)
$$

In (3.1), the number $\left(i_{1}, \ldots, i_{s}\right)$, where $s \leq n-1$ is not fixed, with $0 \leq i_{1} \leq \cdots \leq i_{s} \leq 2^{m-2}$ is $\left(\begin{array}{c}n+2^{m-2} \\ n-1\end{array}\right)$, which is the number of " $2^{m-2}+2$ choose $n-1$ with repetition". When $i_{1}, \ldots, i_{s}$ are chosen, the number of choices for $(\tau, e)$ in $(3.1)$ is $2^{m-1}$. So,

$$
\left|\left(\bigcup_{\substack{1 \leq \nu \leq m-1 \\
0 \leq \tau<2^{\nu}}} \mathcal{B}_{\nu, \tau}\right) / \sim\right|=2^{m-1}\left(\begin{array}{c}
n+2^{m-2} \\
n-1
\end{array}\right) .
$$

In (3.2), for each $0 \leq \nu \leq m-2$, the number of $\left(i_{1}, \ldots, i_{s} ; t\right)$, where $s \leq n-2$ is not fixed, with $0 \leq i_{1} \leq \cdots \leq i_{s} \leq 2^{m-2}, \min \left\{\nu_{2}\left(i_{1}\right), \ldots, \nu_{2}\left(i_{s}\right)\right\} \geq \nu$ and $1 \leq t \leq n-s-1$ is $\left(\begin{array}{c}n+2^{m-2-\nu} \\ n-2\end{array}\right)$, which is the number of " $2^{m-2-\nu}+3$ choose $n-2$ with repetition". When $\nu$ and $\left(i_{1}, \ldots, i_{s} ; t\right)$ are chosen, the number of choices for $(\tau, e)$ in $(3.2)$ is $2^{m-2}$. So,

$$
\left|\left(\bigcup_{\substack{0 \leq \nu \leq m-2 \\
0 \leq \tau<2^{\nu}}} \mathcal{C}_{\nu, \tau}\right) / \sim\right|=2^{m-2} \sum_{\nu=0}^{m-2}\left(\begin{array}{c}
n+2^{m-2-\nu} \\
n-2
\end{array}\right)
$$

Therefore,

$$
\begin{aligned}
\left|Q_{2^{m}}^{n} / \sim\right| & =|\mathcal{A} / \sim|+\left|\left(\bigcup_{\substack{1 \leq \nu \leq m-1 \\
0 \leq \tau<2^{\nu}}} \mathcal{B}_{\nu, \tau}\right) / \sim\right|+\left|\left(\underset{\substack{0 \leq \nu \leq m-2 \\
0 \leq \tau<2^{\nu}}}{\bigcup} \mathcal{C}_{\nu, \tau}\right) / \sim\right| \\
& =\left(\begin{array}{c}
n+2^{m-1}-1 \\
n
\end{array}\right)+2^{m-1}\left(\begin{array}{c}
n+2^{m-2} \\
n-1
\end{array}\right)+2^{m-2} \sum_{\nu=0}^{m-2}\left(\begin{array}{c}
n+2^{m-2-\nu} \\
n-2
\end{array}\right) .
\end{aligned}
$$


Corollary 3.4. (i) Let $n_{0}, \ldots, n_{2^{m-1}-1} \in \mathbb{N}$ such that $n_{0}+\cdots+n_{2^{m-1}-1}=n$. Then

$$
\mid[\underbrace{\alpha^{0}, \ldots, \alpha^{0}}_{n_{0}}, \ldots, \underbrace{\alpha^{2^{m-1}-1}, \ldots, \alpha^{2^{m-1}-1}}_{n_{2^{m-1}-1}} \mid=\left(\begin{array}{c}
n \\
n_{0}, \ldots, n_{2^{m-1}-1}
\end{array}\right) \text {. }
$$

(ii) Let $1 \leq \nu \leq m-1,0 \leq \tau<2^{\nu}$, and $0 \leq e<2^{m-1-\nu}$. Let $n_{0}, \ldots, n_{2^{m-2}} \in \mathbb{N}$ such that $n_{0}+\cdots+n_{2^{m-2}} \leq n-1$ and $\min \left(\left\{\nu_{2}(i): n_{i}>0\right\} \cup\{m-2\}\right)=\nu-1$. Then

$$
\begin{aligned}
& \left|[\underbrace{\alpha^{0}, \ldots, \alpha^{0}}_{n_{0}}, \ldots, \underbrace{\alpha^{2^{m-2}}, \ldots, \alpha^{2^{m-2}}}_{n_{2^{m-2}}}, \alpha^{\tau+2^{\nu} e} \beta, \alpha^{\tau} \beta, \ldots, \alpha^{\tau} \beta]\right| \\
& =\left(\begin{array}{c}
n \\
n_{0}, \ldots, n_{2^{m-2}}, n-n_{0}-\cdots-n_{2^{m-2}}
\end{array}\right) 2^{(m-1-\nu)\left(n-n_{0}-\cdots-n_{\left.2^{m-2}-1\right)+n_{0}+\cdots+n_{2^{m-2}-1}} .\right.}
\end{aligned}
$$

(iii) Let $0 \leq \nu \leq m-2,0 \leq \tau<2^{\nu}$, and $0 \leq e<2^{m-1-\nu}$, e $\equiv 1(\bmod 2)$. Let $n_{0}, \ldots, n_{2^{m-2}} \in \mathbb{N}$ and $t>0$ such that $n_{0}+\cdots+n_{2^{m-2}}+t \leq n-1$ and $\min \left(\left\{\nu_{2}(i)\right.\right.$ : $\left.\left.n_{i}>0\right\} \cup\{m-2\}\right) \geq \nu$. Then

$$
\begin{aligned}
& \left|[\underbrace{\alpha^{0}, \ldots, \alpha^{0}}_{n_{0}}, \ldots, \underbrace{\alpha^{2^{m-2}}, \ldots, \alpha^{2^{m-2}}}_{n_{2^{m-2}}}, \alpha^{\tau+2^{\nu} e} \beta, \alpha^{\tau+2^{\nu}} \beta, \ldots, \alpha^{\tau+2^{\nu}} \beta, \underbrace{\alpha^{\tau} \beta, \ldots, \alpha^{\tau} \beta}_{t}]\right| \\
= & \left(\begin{array}{c}
n \\
n_{0}, \ldots, n_{2^{m-2}}, t, n-n_{0}-\cdots-n_{2^{m-2}}-t
\end{array}\right) 2^{(m-2-\nu)\left(n-n_{0}-\cdots-n_{2^{m-2}}-1\right)+n_{0}+\cdots+n_{2^{m-2}-1}} .
\end{aligned}
$$

Proof. The formulas follow from Corollary 3.2 and simple counting arguments.

\section{$4 B_{n}$-orbits in Tuples of Dihedral Groups}

The dihedral group $D_{N}$ of order $2 N$ is given by the presentation

$$
D_{N}=\left\langle\alpha \beta \mid \alpha^{N}=1=\beta^{2}, \beta \alpha \beta^{-1}=\alpha^{-1}\right\rangle .
$$

Each element of $D_{N}$ can be uniquely written as $\alpha^{i} \beta^{j}$ with $0 \leq i<N$ and $0 \leq j \leq 1$. Clearly, equations (2.1) and (2.2), hence (2.3) - (2.6), also hold for $D_{N}$. In these equations, the only difference between $D_{N}$ and $Q_{2^{m}}$ that affects the Hurwitz action is that $o(\alpha)=N$ in $D_{N}$ but $o(\alpha)=2^{m-1}$ in $Q_{2^{m}}$. When $N=2^{m-1}$, there is no difference. Therefore, under the bijection $D_{2^{m-1}} \rightarrow Q_{2^{m}}, \alpha^{i} \beta^{j} \mapsto \alpha^{i} \beta^{j}, 0 \leq i<2^{m-1}, 0 \leq j \leq 1$, the action of $B_{n}$ on $D_{2^{m-1}}^{n}$ is identical to that on $Q_{2^{m}}^{n}$. Hence, all results in section 3 hold with $Q_{2^{m}}$ replaced by $D_{2^{m-1}}$.

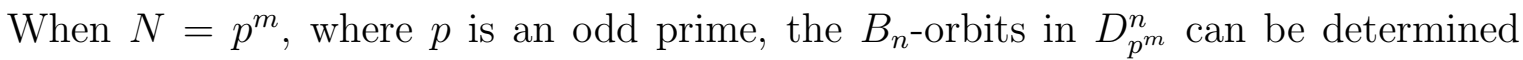
using a method similar to that of section 3 .

For $\boldsymbol{a}=\left(\alpha^{i_{1}} \beta^{j_{1}}, \ldots, \alpha^{i_{n}} \beta^{j_{n}}\right) \in D_{p^{m}}^{n}$, where $0 \leq i_{k}<p^{m}$ and $0 \leq j_{k} \leq 1$, let

$$
\begin{gathered}
\lambda(\boldsymbol{a})=\text { the multi set }\left\{\min \left\{i_{k}, p^{m}-i_{k}\right\}: j_{k}=0\right\}, \\
\gamma(\boldsymbol{a})=\left\{i_{k}: j_{k}=1\right\} .
\end{gathered}
$$


$\lambda(\boldsymbol{a})$ is an invariant of the Hurwitz action on $D_{p^{m}}^{n}$. Let

$$
\mathfrak{A}=\left\{\boldsymbol{a} \in D_{p^{m}}^{n}: \gamma(\boldsymbol{a})=\varnothing\right\} .
$$

Moreover, for $0 \leq \nu \leq m$ and $0 \leq \tau<p^{\nu}$, let

$$
\mathfrak{B}_{\nu, \tau}=\left\{\boldsymbol{a} \in D_{p^{m}}^{n}: \min \left(\left\{\nu_{p}(i): i \in \lambda(\boldsymbol{a})\right\} \cup\{m\}\right)=\nu, \varnothing \neq \gamma(\boldsymbol{a}) \subset \tau+p^{\nu} \mathbb{Z}\right\} ;
$$

for $0 \leq \nu \leq m-1$ and $0 \leq \tau<p^{\nu}$, let

$$
\begin{gathered}
\mathfrak{C}_{\nu, \tau}=\left\{\boldsymbol{a} \in D_{p^{m}}^{n}: \min \left(\left\{\nu_{p}(i): i \in \lambda(\boldsymbol{a})\right\} \cup\{m\}\right) \geq \nu+1, \varnothing \neq \gamma(\boldsymbol{a}) \subset \tau+p^{\nu} \mathbb{Z},\right. \\
\left.\exists j, j^{\prime} \in \gamma(\boldsymbol{a}) \text { such that } \nu_{p}\left(j-j^{\prime}\right)=\nu\right\} .
\end{gathered}
$$

Then $\mathfrak{A}, \mathfrak{B}_{\nu, \tau}$ and $\mathfrak{C}_{\nu, \tau}$ are all invariant under the Hurwitz equivalence and

$$
D_{p^{m}}=\mathfrak{A} \dot{\cup}\left(\bigcup_{\substack{0 \leq \nu \leq m \\ 0 \leq \tau<p^{\nu}}}^{\dot{B}} \mathfrak{B}_{\nu, \tau}\right) \dot{\cup}\left(\bigcup_{\substack{0 \leq \nu \leq m-1 \\ 0 \leq \tau<p^{\nu}}} \mathfrak{C}_{\nu, \tau}\right) .
$$

For $\boldsymbol{a} \in \mathfrak{C}_{\nu, \tau}$, collect the components of $\boldsymbol{a}$ of the form $\alpha^{i} \beta$ and let the result be $\left(\alpha^{i_{1}} \beta, \ldots, \alpha^{i_{t}} \beta\right)$, where $0 \leq i_{k}<p^{m}$. Let $e_{k} \in \mathbb{Z}_{p}, 1 \leq k \leq t$, be defined by $i_{k} \equiv \tau+p^{\nu} e_{k}$ $\left(\bmod p^{\nu+1}\right)$. Put

$$
\sigma(\boldsymbol{a})=\sum_{k=1}^{t}(-1)^{k-1} e_{k}
$$

For example, let $p=5, m=4, n=5$, and let

$$
\boldsymbol{a}=\left(\alpha^{9+5^{2} \cdot 4} \beta, \alpha^{5^{3} \cdot 3}, \alpha^{9+5^{2} \cdot 2} \beta, \alpha^{9+5^{2} \cdot 8} \beta, \alpha^{9+5^{2}} \beta\right) \in \mathfrak{C}_{2,9} .
$$

Then $\sigma(\boldsymbol{a})=4-2+8-1=4 \in \mathbb{Z}_{5}$. From (2.3) - (2.6), it is easy to see that $\sigma(\boldsymbol{a})$ is an invariant under the Hurwitz equivalence. Further partition $\mathfrak{C}_{\nu, \tau}$ as

$$
\mathfrak{C}_{\nu, \tau}^{0}=\left\{\boldsymbol{a} \in \mathfrak{C}_{\nu, \tau}^{\mathfrak{r}}: \sigma(\boldsymbol{a})=0\right\}
$$

and

$$
\mathfrak{C}_{\nu, \tau}^{1}=\left\{\boldsymbol{a} \in \mathfrak{C}_{\nu, \tau}: \sigma(\boldsymbol{a}) \neq 0\right\} .
$$

Lemma 4.1. Let $\tau, \nu, e, f \in \mathbb{Z}$ such that $0 \leq \nu \leq m-1$ and $e \not \equiv f(\bmod p)$. Then for every $g \in \mathbb{Z}$,

$$
\left(\alpha^{\tau+p^{\nu} e} \beta, \alpha^{\tau+p^{\nu} f} \beta\right) \sim\left(\alpha^{\tau+p^{\nu}(e+g)} \beta, \alpha^{\tau+p^{\nu}(f+g)} \beta\right) .
$$

The proof of Lemma 4.1 is the same as that of Lemma 2.1 (iii).

Theorem 4.2. $\quad$ (i) The $B_{n}$-orbits in $\mathfrak{A}$ are represented by

$$
\left(\alpha^{i_{1}}, \ldots, \alpha^{i_{n}}\right),
$$

where $0 \leq i_{1} \leq \cdots \leq i_{n}<p^{m}$. 
(ii) Let $0 \leq \nu \leq m$ and $0 \leq \tau<p^{\nu}$. The $B_{n}$-orbits in $\mathfrak{B}_{\nu, \tau}$ are represented by

$$
\left(\alpha^{i_{1}}, \ldots, \alpha^{i_{s}}, \alpha^{\tau+p^{\nu} e} \beta, \alpha^{\tau} \beta, \ldots, \alpha^{\tau} \beta\right),
$$

where $0 \leq i_{1} \leq \cdots \leq i_{s}<\frac{1}{2} p^{m}, \min \left\{\nu_{p}\left(i_{1}\right), \ldots, \nu_{p}\left(i_{s}\right), m\right\}=\nu, 0 \leq e<p^{m-\nu}$.

(iii) Let $0 \leq \nu \leq m-1$ and $0 \leq \tau<p^{\nu}$.

(iii-1) The $B_{n}$-orbits in $\mathfrak{C}_{\nu, \tau}^{0}$ are represented by

$$
(\alpha^{i_{1}}, \ldots, \alpha^{i_{s}}, \alpha^{\tau+p^{\nu} e} \beta, \alpha^{\tau+p^{\nu}} \beta, \underbrace{\alpha^{\tau} \beta, \ldots, \alpha^{\tau} \beta}_{n-2-s})
$$

where $0 \leq i_{1} \leq \cdots \leq i_{s}<\frac{1}{2} p^{m}, n-2-s>0, \min \left\{\nu_{p}\left(i_{1}\right), \ldots, \nu_{p}\left(i_{s}\right), m\right\} \geq \nu+1$, $0 \leq e<p^{m-\nu}, e \equiv 1(\bmod p)$.

(iii-2) The $B_{n}$-orbits in $\mathfrak{C}_{\nu, \tau}^{1}$ are represented by

$$
(\alpha^{i_{1}}, \ldots, \alpha^{i_{s}}, \alpha^{\tau+p^{\nu} e} \beta, \underbrace{\alpha^{\tau} \beta, \ldots, \alpha^{\tau} \beta}_{n-1-s}),
$$

where $0 \leq i_{1} \leq \cdots \leq i_{s}<\frac{1}{2} p^{m}, n-1-s>0, \min \left\{\nu_{p}\left(i_{1}\right), \ldots, \nu_{p}\left(i_{s}\right), m\right\} \geq \nu+1$, $0 \leq e<p^{m-\nu}, e \not \equiv 0(\bmod p)$.

Proof. The proofs of (i) and (ii) are identical to those of the corresponding cases in Theorem 3.1.

(iii) Different tuples in (4.1) are nonequivalent since they have different combinations of invariants $\lambda(\boldsymbol{a})$ and $\pi(\boldsymbol{a})$. The same is true for the tuples in (4.2). Therefore, it remains to show that every tuple $\boldsymbol{a} \in \mathfrak{C}_{\nu, \tau}$ is equivalent to one of the tuples in (4.1) or (4.2).

By $(2.3)-(2.5)$, we may write

$$
\boldsymbol{a}=\left(\alpha^{i_{1}}, \ldots, \alpha^{i_{s}}, \alpha^{\tau+p^{\nu} e_{1}} \beta, \ldots, \alpha^{\tau+p^{\nu} e_{t}} \beta\right)
$$

where $0 \leq i_{1} \leq \cdots \leq i_{s}<\frac{1}{2} p^{m}$ and there exist $k, l$ such that $e_{k} \not \equiv e_{l}(\bmod p)$. It suffices to show that either

$$
\left(\alpha^{\tau+p^{\nu} e_{1}} \beta, \ldots, \alpha^{\tau+p^{\nu} e_{t}} \beta\right) \sim\left(\alpha^{\tau+p^{\nu} e} \beta, \alpha^{\tau+p^{\nu}} \beta, \alpha^{\tau} \beta, \ldots, \alpha^{\tau} \beta\right)
$$

for some $0 \leq e<p^{m-\nu}$ with $e \equiv 1(\bmod p)$ or

$$
\left(\alpha^{\tau+p^{\nu} e_{1}} \beta, \ldots, \alpha^{\tau+p^{\nu} e_{t}} \beta\right) \sim\left(\alpha^{\tau+p^{\nu} e} \beta, \alpha^{\tau} \beta, \ldots, \alpha^{\tau} \beta\right)
$$

for some $0 \leq e<p^{m-\nu}$ with $e \not \equiv 0(\bmod p)$. We prove this claim by induction on $t$.

If $t=2$, by Lemma 4.1, we have

$$
\left(\alpha^{\tau+p^{\nu} e_{1}} \beta, \alpha^{\tau+p^{\nu} e_{2}} \beta\right) \sim\left(\alpha^{\tau+p^{\nu}\left(e_{1}-e_{2}\right)} \beta, \alpha^{\tau} \beta\right)
$$

hence (4.4) holds. 
Now assume $t>2$. Assume that $e_{k} \not \equiv e_{k+1} \equiv \cdots \equiv e_{t}(\bmod p)$. By Lemma 4.1,

$$
\begin{aligned}
& \left(\alpha^{\tau+p^{\nu} e_{k}} \beta, \alpha^{\tau+p^{\nu} e_{k+1}} \beta, \ldots, \alpha^{\tau+p^{\nu} e_{t}} \beta\right) \\
\sim & \left(\alpha^{\tau+p^{\nu} e_{k}^{\prime}} \beta, \alpha^{\tau+p^{\nu} e_{k}} \beta, \ldots, \alpha^{\tau+p^{\nu} e_{t}} \beta\right) \\
\sim & \cdots \\
\sim & \left(\alpha^{\tau+p^{\nu} e_{k}^{\prime}} \beta, \cdots, \alpha^{\tau+p^{\nu} e_{t-2}^{\prime}} \beta, \alpha^{\tau+p^{\nu} e_{k}} \beta, \alpha^{\tau+p^{\nu} e_{t}} \beta\right) \\
\sim & \left(\alpha^{\tau+p^{\nu} e_{k}^{\prime}} \beta, \cdots, \alpha^{\tau+p^{\nu} e_{t-1}^{\prime}} \beta, \alpha^{\tau} \beta\right) .
\end{aligned}
$$

So,

$$
\left(\alpha^{\tau+p^{\nu} e_{1}} \beta, \cdots, \alpha^{\tau+p^{\nu} e_{t}} \beta\right) \sim\left(\alpha^{\tau+p^{\nu} f_{1}} \beta, \cdots, \alpha^{\tau+p^{\nu} f_{t-1}} \beta, \alpha^{\tau} \beta\right) .
$$

If $f_{1}, \ldots, f_{t-1}$ are not all the same modulo $p$, the induction hypothesis applies to $\left(\alpha^{\tau+p^{\nu} f_{1}} \beta, \cdots, \alpha^{\tau+p^{\nu} f_{t-1}} \beta\right)$. So, assume $f_{1} \equiv \cdots \equiv f_{t-1} \not \equiv 0(\bmod p)$. Let $x \in \mathbb{Z}$ such that $x \not \equiv-f_{t-1}(\bmod p)$. Then

$$
\begin{aligned}
& \left(\alpha^{\tau+p^{\nu} f_{t-2}} \beta, \alpha^{\tau+p^{\nu} f_{t-1}} \beta, \alpha^{\tau} \beta\right) \\
\sim & \left(\alpha^{\tau+p^{\nu} f_{t-2}} \beta, \alpha^{\tau+p^{\nu}\left(f_{t-1}+1\right)} \beta, \alpha^{\tau+p^{\nu}} \beta\right) \\
\sim & \left(\alpha^{\tau+p^{\nu}\left(f_{t-2}+x\right)} \beta, \alpha^{\tau+p^{\nu}\left(f_{t-1}+x+1\right)} \beta, \alpha^{\tau+p^{\nu}} \beta\right) \\
\sim & \left(\alpha^{\tau+p^{\nu}\left(f_{t-2}+x\right)} \beta, \alpha^{\tau+p^{\nu}\left(f_{t-1}+x\right)} \beta, \alpha^{\tau} \beta\right) .
\end{aligned}
$$

If $t=3$, choose $x=-f_{t-1}+1$, then (4.4) holds. If $t>3$, choose $x \in \mathbb{Z}$ such that $x \not \equiv-f_{t-1}, 0(\bmod p)$. Then the induction hypothesis applies to

$$
\left(\alpha^{\tau+p^{\nu} f_{1}} \beta, \cdots, \alpha^{\tau+p^{\nu} f_{t-3}} \beta, \alpha^{\tau+p^{\nu}\left(f_{t-2}+x\right)} \beta, \alpha^{\tau+p^{\nu}\left(f_{t-1}+x\right)} \beta\right) .
$$

Corollary 4.3. (i) $\boldsymbol{a}, \boldsymbol{b} \in \mathfrak{A}$ are equivalent $\Leftrightarrow \boldsymbol{a}$ is a permutation of $\boldsymbol{b}$.

(ii) $\boldsymbol{a}, \boldsymbol{b} \in \mathfrak{B}_{\nu, \tau}$ are equivalent $\Leftrightarrow \lambda(\boldsymbol{a})=\lambda(\boldsymbol{b})$ and $\pi(\boldsymbol{a})=\pi(\boldsymbol{b})$.

(iii) $\boldsymbol{a}, \boldsymbol{b} \in \mathfrak{C}_{\nu, \tau}$ are equivalent $\Leftrightarrow \lambda(\boldsymbol{a})=\lambda(\boldsymbol{b}), \sigma(\boldsymbol{a})=\sigma(\boldsymbol{b})$ and $\pi(\boldsymbol{a})=\pi(\boldsymbol{b})$.

We remark that the $B_{n^{-}}$orbits of $D_{2 p^{m}}^{n}$, where $p$ is an odd prime, can also be determined due to the fact that $D_{2 p^{m}} \cong \mathbb{Z}_{2} \times D_{p^{m}}$. However, for an arbitrary positive integer $N$, determination of the $B_{n}$-orbits of $D_{N}^{n}$ seems to be a difficult problem.

Acknowledgment: The author thanks the referee for pointing out an error in Corollary 3.3 in a previous version of the paper.

\section{References}

[1] T. Ben-Itzhak and M. Teicher, Graph theoretic method for determining Hurwitz equivalence in the symmetric group, Israel J. Math. 135 (2003), 83 - 91.

[2] S. P. Humphries, Finite Hurwitz braid group actions on sequences of Euclidean reflections, J. Algebra 269 (2003), 556 - 58.

[3] J. Michel, Hurwitz action on tuples of Euclidean reflections, J. Algebra 295 (2006), $289-292$. 